\title{
Highly Sensitive Near-Infrared Fluorescent Probes for Nitric Oxide and Their Application to Isolated Organs
}

\author{
Eita Sasaki, ${ }^{\dagger}$ Hirotatsu Kojima, ${ }^{\dagger}$ Hiroaki Nishimatsu, ${ }^{\ddagger}$ Yasuteru Urano, ${ }^{\dagger}, \xi$ Kazuya Kikuchi, ${ }^{\dagger}, \S$ \\ Yasunobu Hirata, ${ }^{\dagger}$ and Tetsuo Nagano* ${ }^{\dagger} \dagger$ \\ ${ }^{\dagger}$ Graduate School of Pharmaceutical Sciences, and ${ }^{\ddagger}$ Faculty of Medicine, The University of Tokyo, 7-3-1 \\ Hongo, Bunkyo-ku, Tokyo 113-0033, Japan, and ${ }^{\S}$ PRESTO, Japan Science and Technology Corporation, \\ Kawaguchi, Saitama, Japan \\ *E-mail Address: tlong@mol.f.u-tokyo.ac.jp
}

\section{Supporting Information}

General procedures. IR-780 iodide and IR-783 were purchased from Sigma-Aldrich Co. NOC13 was synthesized as described previously. ${ }^{1}$ Its half-life in aqueous buffer solution at $\mathrm{pH} 7.4$ and $37{ }^{\circ} \mathrm{C}$ was determined by following the disappearance of the characteristic UV absorption $\left(\lambda_{\max }=250 \mathrm{~nm}\right)$. All other reagents and solvents were purchased from commercial sources. Reactions were monitored by TLC with visual observation of the dye spots and by mass spectrometry. Products were purified on a silica gel column, and by semi-preparative HPLC. Products were considered pure when each single HPLC peak was obtained. Quantum yields of fluorescence were determined using that of ICG (0.13) in DMSO as a standard. ${ }^{2}$ A saturated NO aqueous solution was prepared by bubbling purified NO gas through deoxygenated buffer. The saturation was ascertained by employing the Griess method ${ }^{3}$ after autoxidation of $\mathrm{NO}$ to nitrite and comparing to the reported solubility of NO in water at room temperature $(2 \mathrm{mM}){ }^{4}$

Structure of ICG (Indocyanine Green)

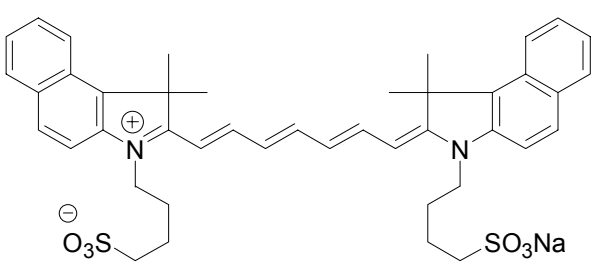

\section{References}

(1) Hrabie, J. A.; Klose, J. R.; Wink, D. A.; Keefer, L. K. J. Org. Chem. 1993, 58, 1472-1476.

(2) Benson, R. C.; Kues, H. A. J. Chem. Eng. Data. 1977, 22, 379-383.

(3) Green, L. C.; Wagner, D. A.; Glogowski, J.; Skipper, P. L.; Wishnok, J. S.; Tannenbaum, S. R. Anal. Biochem. 1982, 126, 131-138.

(4) Dean, J. A. Lange's Handbook of Chemistry 14th ed.; McGraw-Hill, Inc.: New York, 1992; Section $5,5.6$.

Instruments. ${ }^{1} \mathrm{H}-\mathrm{NMR}$ spectra were recorded on a JEOL JNM-LA300. Mass spectra were measured 
with a JEOL JMS-700 mass spectrometer $\left(\mathrm{FAB}^{+}\right)$, a JEOL JMS-T100LC mass spectrometer $\left(\mathrm{ESI}^{+}\right)$, or a JEOL JMS-DX300 $\left(\mathrm{EI}^{+}\right)$. HPLC purification and analyses were performed on a reverse-phase column (GL Sciences (Tokyo, Japan), Inertsil ODS-3 $10 \mathrm{~mm} \times 250 \mathrm{~mm}$ for purification and Inertsil ODS-3 4.6 $\mathrm{mm} \times 250 \mathrm{~mm}$ for analyses) using eluent $\mathrm{A}\left(\mathrm{H}_{2} \mathrm{O}\right.$ with $0.1 \%$ trifluoroacetic acid (TFA)) and eluent $\mathrm{B}$ $\left(\mathrm{CH}_{3} \mathrm{CN}\right.$ with $20 \% \mathrm{H}_{2} \mathrm{O}$ and $0.1 \%$ TFA), fitted on a Jasco PU-1587 system. The absorbance at $780 \mathrm{~nm}$ was monitored for detection. Extinction coefficients were determined using a Shimadzu UV-1600 (Tokyo, Japan). Fluorescence spectroscopic studies were performed with a Hitachi F4500 (Tokyo, Japan). Fluorescence images were captured with a Leica MZ FLIII fluorescence stereomicroscope equipped with a $75 \mathrm{~W}$ xenon light source, a cooled monochrome camera (Leica DC 350FX), a $730 \pm 23 \mathrm{~nm}$ excitation filter (Omega Optical, XF 1097) and a $770 \mathrm{~nm}$ long-pass emission filter (Omega Optical, XF 3115). Light exposure time was $600 \mathrm{~ms}$. Acquired Images were analyzed with a Meta Vue software (Universal Imaging corporation).

\section{Synthetic schemes of DACs and DAC-Ts}

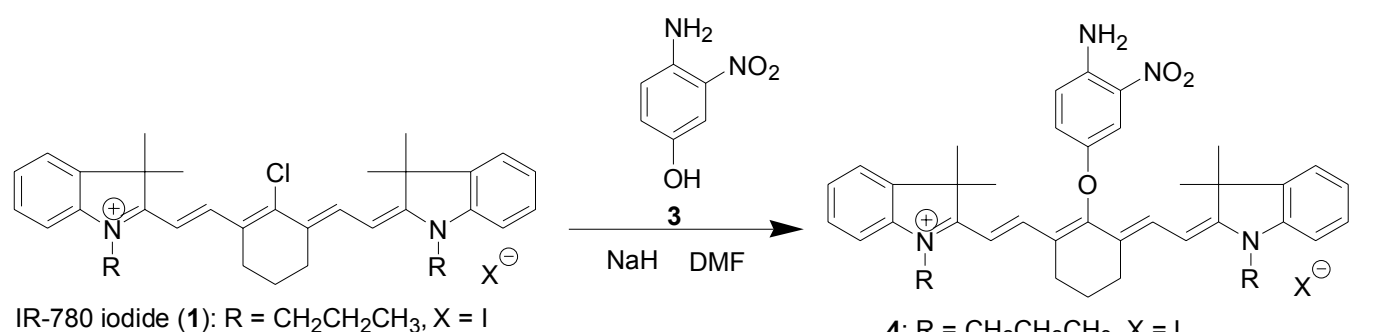

IR-783 (2): $\mathrm{R}=\mathrm{CH}_{2}\left(\mathrm{CH}_{2}\right)_{3} \mathrm{SO}_{3} \mathrm{Na}, \mathrm{CH}_{2}\left(\mathrm{CH}_{2}\right)_{3} \mathrm{SO}_{3}^{-}$

4: $\mathrm{R}=\mathrm{CH}_{2} \mathrm{CH}_{2} \mathrm{CH}_{3}, \mathrm{X}=\mathrm{I}$

5: $\mathrm{R}=\mathrm{CH}_{2}\left(\mathrm{CH}_{2}\right)_{3} \mathrm{SO}_{3} \mathrm{Na}, \mathrm{CH}_{2}\left(\mathrm{CH}_{2}\right)_{3} \mathrm{SO}_{3}{ }^{-}$
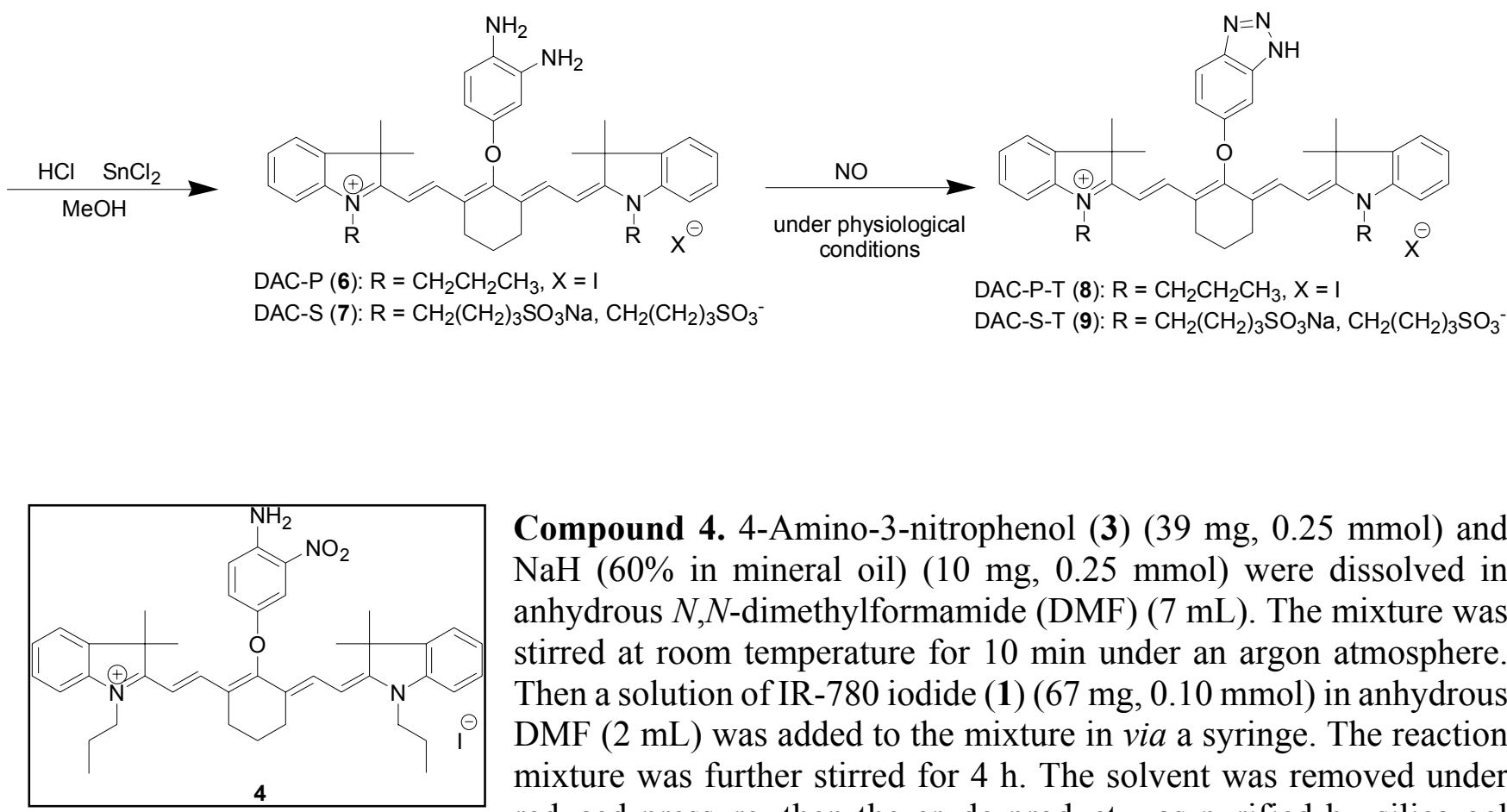

Compound 4. 4-Amino-3-nitrophenol (3) (39 $\mathrm{mg}, 0.25 \mathrm{mmol})$ and $\mathrm{NaH}(60 \%$ in mineral oil) $(10 \mathrm{mg}, 0.25 \mathrm{mmol})$ were dissolved in anhydrous $N, N$-dimethylformamide (DMF) $(7 \mathrm{~mL})$. The mixture was stirred at room temperature for 10 min under an argon atmosphere. Then a solution of IR-780 iodide (1) $(67 \mathrm{mg}, 0.10 \mathrm{mmol})$ in anhydrous DMF $(2 \mathrm{~mL})$ was added to the mixture in via a syringe. The reaction mixture was further stirred for $4 \mathrm{~h}$. The solvent was removed under reduced pressure, then the crude product was purified by silica gel chromatography with 7\% $\mathrm{MeOH}$ in dichloromethane (DCM) to afford the desired product as a dark green solid (57 mg, 72\%). ${ }^{1} \mathrm{H}$ NMR (300 MHz, $\left.\mathrm{CDCl}_{3}\right): \delta 1.05(\mathrm{t}, 6 \mathrm{H}, J=7.3 \mathrm{~Hz}), 1.42(\mathrm{~s}, 12 \mathrm{H}), 1.87(\mathrm{~m}, 4 \mathrm{H})$, $2.04(\mathrm{~m}, 2 \mathrm{H}), 2.68(\mathrm{t}, 4 \mathrm{H}, J=5.0 \mathrm{~Hz}), 3.99(\mathrm{t}, 4 \mathrm{H}, J=7.2 \mathrm{~Hz}), 5.99(\mathrm{~d}, 2 \mathrm{H}, J=14.4 \mathrm{~Hz}), 6.90(\mathrm{~s}, 2 \mathrm{H})$, 7.05-7.40 (m, 9H), 7.66-7.72 (m, 2H), $7.92(\mathrm{~d}, 2 \mathrm{H}, J=14.4 \mathrm{~Hz}) . \mathrm{MS}\left(\mathrm{FAB}^{+}\right): 657\left(\mathrm{M}-\mathrm{I}^{-}\right)$. HRMS $\left(\mathrm{ESI}^{+}\right)$: calcd 657.3753, found $657.3799\left(\mathrm{M}-\mathrm{I}^{-}\right)$. 


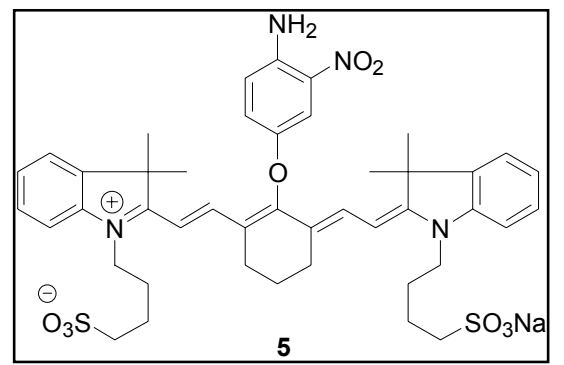

Compound 5. 4-Amino-3-nitrophenol (3) (93 mg, $0.60 \mathrm{mmol}$ ) and $\mathrm{NaH}(60 \%$ in mineral oil) $(24 \mathrm{mg}, 0.60 \mathrm{mmol})$ were dissolved in anhydrous DMF $(16 \mathrm{~mL})$. The mixture was stirred at room temperature for $10 \mathrm{~min}$ under an argon atmosphere. Then a solution of IR-783 (2) (180 mg, $0.24 \mathrm{mmol})$ in anhydrous DMF (5 mL) was added to the mixture in via a syringe. The reaction mixture was further stirred for $4 \mathrm{~h}$. The solvent was removed under reduced pressure, then the crude product was purified by silica gel chromatography with $10 \%$ $\mathrm{H}_{2} \mathrm{O}$ in $\mathrm{CH}_{3} \mathrm{CN}$ to afford the desired product as a dark green solid $(176 \mathrm{mg}, 85 \%)$. ${ }^{1} \mathrm{H} \mathrm{NMR}(300 \mathrm{MHz}$, $\left.\mathrm{CD}_{3} \mathrm{OD}\right): \delta 1.33(\mathrm{~s}, 12 \mathrm{H}), 1.80-1.96(\mathrm{~m}, 10 \mathrm{H}), 2.67(\mathrm{t}, 4 \mathrm{H}, J=5.9 \mathrm{~Hz}), 2.78(\mathrm{t}, 4 \mathrm{H}, J=7.0 \mathrm{~Hz}), 4.04(\mathrm{t}$, $4 \mathrm{H}, J=6.3 \mathrm{~Hz}), 6.12(\mathrm{~d}, 2 \mathrm{H}, J=14.3 \mathrm{~Hz}), 6.97(\mathrm{~d}, 1 \mathrm{H}, J=9.3 \mathrm{~Hz}), 7.08-7.31(\mathrm{~m}, 9 \mathrm{H}), 7.61(\mathrm{~d}, 1 \mathrm{H}, J=2.9$ $\mathrm{Hz}), 7.89(\mathrm{~d}, 2 \mathrm{H}, J=14.3 \mathrm{~Hz})$. MS $\left(\mathrm{FAB}^{+}\right): 845\left(\mathrm{M}-\mathrm{Na}^{+}+2 \mathrm{H}^{+}\right), 867\left(\mathrm{M}+\mathrm{H}^{+}\right), 889\left(\mathrm{M}+\mathrm{Na}^{+}\right)$. HRMS $\left(\mathrm{ESI}^{+}\right)$: calcd 889.2893, found 889.2916 $\left(\mathrm{M}+\mathrm{Na}^{+}\right)$.

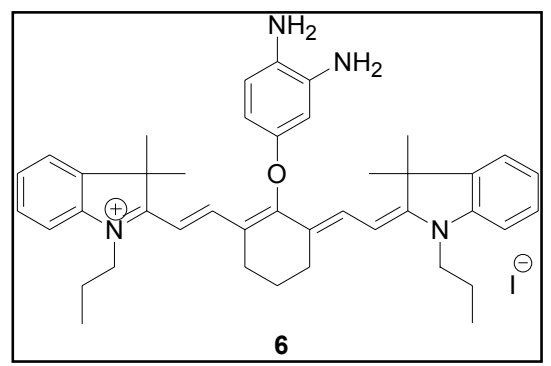

DAC-P (6). To a solution of 4 ( $88 \mathrm{mg}, 0.11 \mathrm{mmol})$ in $\mathrm{MeOH}(4$ $\mathrm{mL})$ and concentrated $\mathrm{HCl}(0.6 \mathrm{~mL})$ was added $\mathrm{SnCl}_{2}-\mathrm{H}_{2} \mathrm{O}(500 \mathrm{mg}$, $2.2 \mathrm{mmol})$. The solution was stirred at room temperature overnight under an argon atmosphere, then neutralized with $2 \mathrm{~N} \mathrm{NaOH}$. The precipitate was removed by filtration and washed with DCM. The filtrate and washing were extracted with DCM and the organic phase was washed with water. The organic extract was dried over $\mathrm{Na}_{2} \mathrm{SO}_{4}$, filtered and evaporated to give a dark green solid (17 mg, 20\%). Pure dye was obtained by semi-preparative HPLC. mp $112-115{ }^{\circ} \mathrm{C} .{ }^{1} \mathrm{H} \mathrm{NMR}\left(300 \mathrm{MHz}, \mathrm{CDCl}_{3}\right): \delta 1.04(\mathrm{t}, 6 \mathrm{H}$, $J=7.3 \mathrm{~Hz}), 1.41(\mathrm{~s}, 12 \mathrm{H}), 1.86(\mathrm{~m}, 4 \mathrm{H}), 2.04$ (quin, $2 \mathrm{H}, J=6.0 \mathrm{~Hz}), 2.71(\mathrm{t}, 4 \mathrm{H}, J=6.0 \mathrm{~Hz}), 4.01(\mathrm{t}, 4 \mathrm{H}$, $J=7.2 \mathrm{~Hz}), 6.02(\mathrm{~d}, 2 \mathrm{H}, J=14.1 \mathrm{~Hz}), 6.34(\mathrm{dd}, 1 \mathrm{H}, J=8.3 \mathrm{~Hz}, 2.8 \mathrm{~Hz}), 6.52(\mathrm{~d}, 1 \mathrm{H}, 2.8 \mathrm{~Hz}), 6.64(\mathrm{~d}, 1 \mathrm{H}$, $8.3 \mathrm{~Hz}), 7.05(\mathrm{~d}, 2 \mathrm{H}, 7.9 \mathrm{~Hz}), 7.15-7.36(\mathrm{~m}, 6 \mathrm{H}), 7.99(\mathrm{~d}, 2 \mathrm{H}, J=14.1 \mathrm{~Hz}) . \mathrm{HRMS}_{\left(\mathrm{ESI}^{+}\right) \text {: calcd }}$ 627.4063, found 627.4037 $\left(\mathrm{M}-\mathrm{I}^{-}\right)$. HPLC (eluent A/B = 35/65-15 min-20/80): $10.51 \mathrm{~min}$.
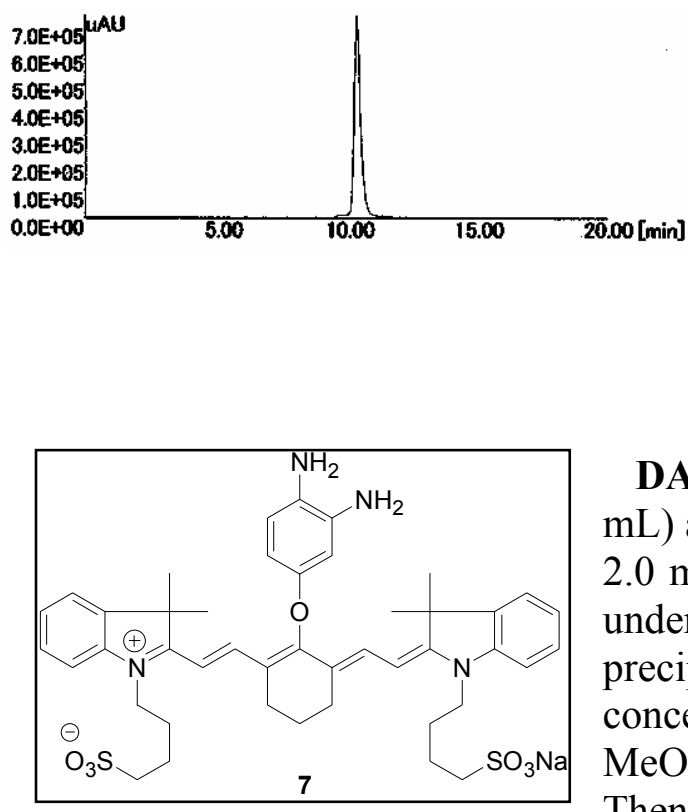

DAC-S (7). To a solution of 5 (100 $\mathrm{mg}, 0.12 \mathrm{mmol})$ in $\mathrm{MeOH}(4$ $\mathrm{mL})$ and concentrated $\mathrm{HCl}(0.6 \mathrm{~mL})$ was added $\mathrm{SnCl}_{2}-\mathrm{H}_{2} \mathrm{O}(450 \mathrm{mg}$, $2.0 \mathrm{mmol})$. The solution was stirred at room temperature overnight under an argon atmosphere, then neutralized with $2 \mathrm{~N} \mathrm{NaOH}$. The precipitate was removed by filtration and the filtrate was concentrated under reduced pressure. The residue was dissolved in $\mathrm{MeOH}(10 \mathrm{~mL})$ and the insoluble salt was removed by filtration. Then the solvent was removed under reduced pressure and the crude product was purified by silica gel chromatography with $8 \% \mathrm{H}_{2} \mathrm{O}$ in $\mathrm{CH}_{3} \mathrm{CN}$ to afford the desired product as a dark green solid (20 mg, 20\%). Further purification was performed by semi-preparative HPLC to 
afford a gold solid. mp 222-224 ${ }^{\circ} \mathrm{C} .{ }^{1} \mathrm{H}$ NMR (300 MHz, $\left.\mathrm{CD}_{3} \mathrm{OD}\right): \delta 1.30(\mathrm{~s}, 12 \mathrm{H}), 1.82-1.92(\mathrm{~m}, 10 \mathrm{H})$, $2.62(\mathrm{t}, 4 \mathrm{H}, J=5.8 \mathrm{~Hz}), 2.77(\mathrm{t}, 4 \mathrm{H}, J=6.7 \mathrm{~Hz}), 4.01(\mathrm{t}, 4 \mathrm{H}, J=5.5 \mathrm{~Hz}), 6.05(\mathrm{~d}, 2 \mathrm{H}, J=14.2 \mathrm{~Hz}), 6.19$ $(\mathrm{dd}, 1 \mathrm{H}, J=8.4,2.9 \mathrm{~Hz}), 6.45(\mathrm{~d}, 1 \mathrm{H}, J=2.9 \mathrm{~Hz}), 6.58(\mathrm{~d}, 1 \mathrm{H}, J=8.4 \mathrm{~Hz}), 7.06-7.28(\mathrm{~m}, 8 \mathrm{H}), 7.97(\mathrm{~d}$, $2 \mathrm{H}, J=14.2 \mathrm{~Hz})$. MS $\left(\mathrm{FAB}^{+}\right): 815\left(\mathrm{M}-\mathrm{Na}^{+}+2 \mathrm{H}^{+}\right), 837\left(\mathrm{M}+\mathrm{H}^{+}\right), 859\left(\mathrm{M}+\mathrm{Na}^{+}\right)$. HRMS $\left(\mathrm{ESI}^{+}\right)$: calcd 837.3332, found 837.3319 $\left(\mathrm{M}+\mathrm{H}^{+}\right)$. HPLC (eluent $\left.\mathrm{A} / \mathrm{B}=54 / 46\right)$ : $12.2 \mathrm{~min}$.
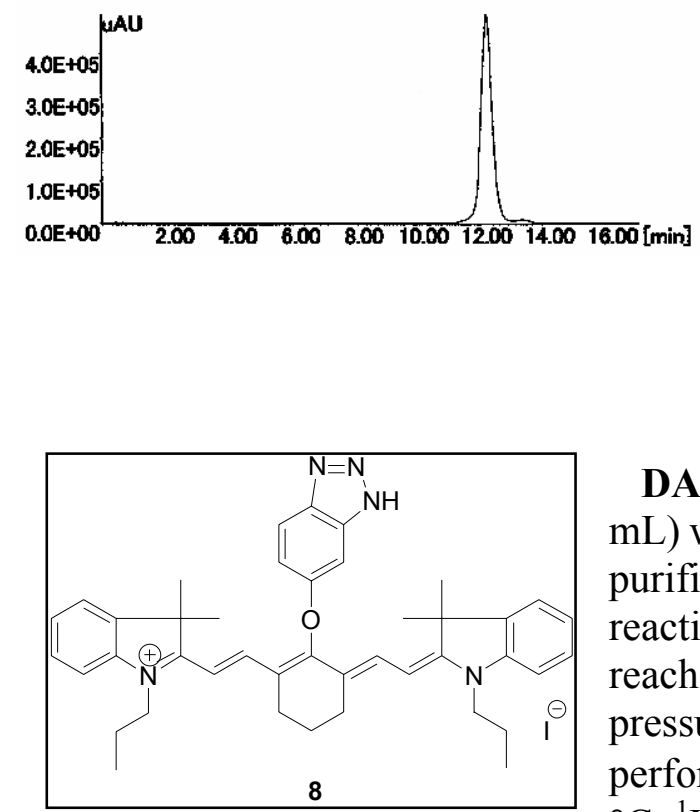

DAC-P-T (8). To a solution of 6 (10 mg, $13 \mu \mathrm{mol})$ in $\mathrm{MeOH}(13$ $\mathrm{mL}$ ) was added $\mathrm{NO}-$ bubbled $\mathrm{MeOH}$, which was prepared by bubbling purified $\mathrm{NO}$ gas through deoxygenated $\mathrm{MeOH}$. The end of the reaction was determined when the fluorescence intensity at $785 \mathrm{~nm}$ reached a maximum. Then the solvent was removed under reduced pressure to afford the desired product. Further purification was performed with semi-preparative HPLC (2.6 mg, 26\%). mp 135-137 ${ }^{\circ} \mathrm{C} .{ }^{1} \mathrm{H}$ NMR $\left(300 \mathrm{MHz}, \mathrm{CDCl}_{3}\right): \delta 1.03(\mathrm{t}, 6 \mathrm{H}, J=7.4 \mathrm{~Hz}), 1.24(\mathrm{~s}$, 12H) $1.84(\mathrm{~m}, 4 \mathrm{H}), 2.07$ (quin, $2 \mathrm{H}, J=5.9 \mathrm{~Hz}), 2.70(\mathrm{t}, 4 \mathrm{H}, J=5.9 \mathrm{~Hz}), 3.90(\mathrm{t}, 4 \mathrm{H}, J=7.3 \mathrm{~Hz}), 5.94(\mathrm{~d}$, $2 \mathrm{H}, 14.2 \mathrm{~Hz}), 7.00$ (d, 2H, 7.9 Hz), 7.14-7.35 (m, 10H), 7.97 (d, 2H, $J=14.2 \mathrm{~Hz}), 8.08(\mathrm{~d}, 1 \mathrm{H}, J=8.6 \mathrm{~Hz})$. HRMS (ESI ${ }^{+}$): calcd 638.3859, found 638.3824 (M - I'). HPLC (eluent A/B = 15/85): 12.5 min.
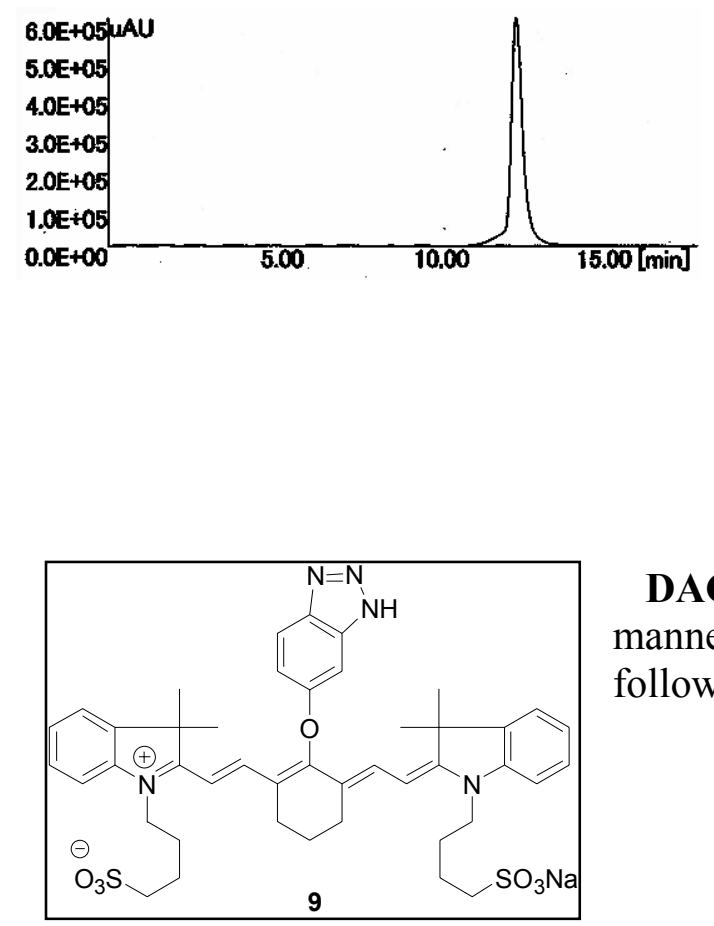

DAC-S-T (9). Compound 9 was prepared from 7 in the same manner as described for 8. Another sample was synthesized as follows:

\section{Synthetic scheme of DAC-S-T}



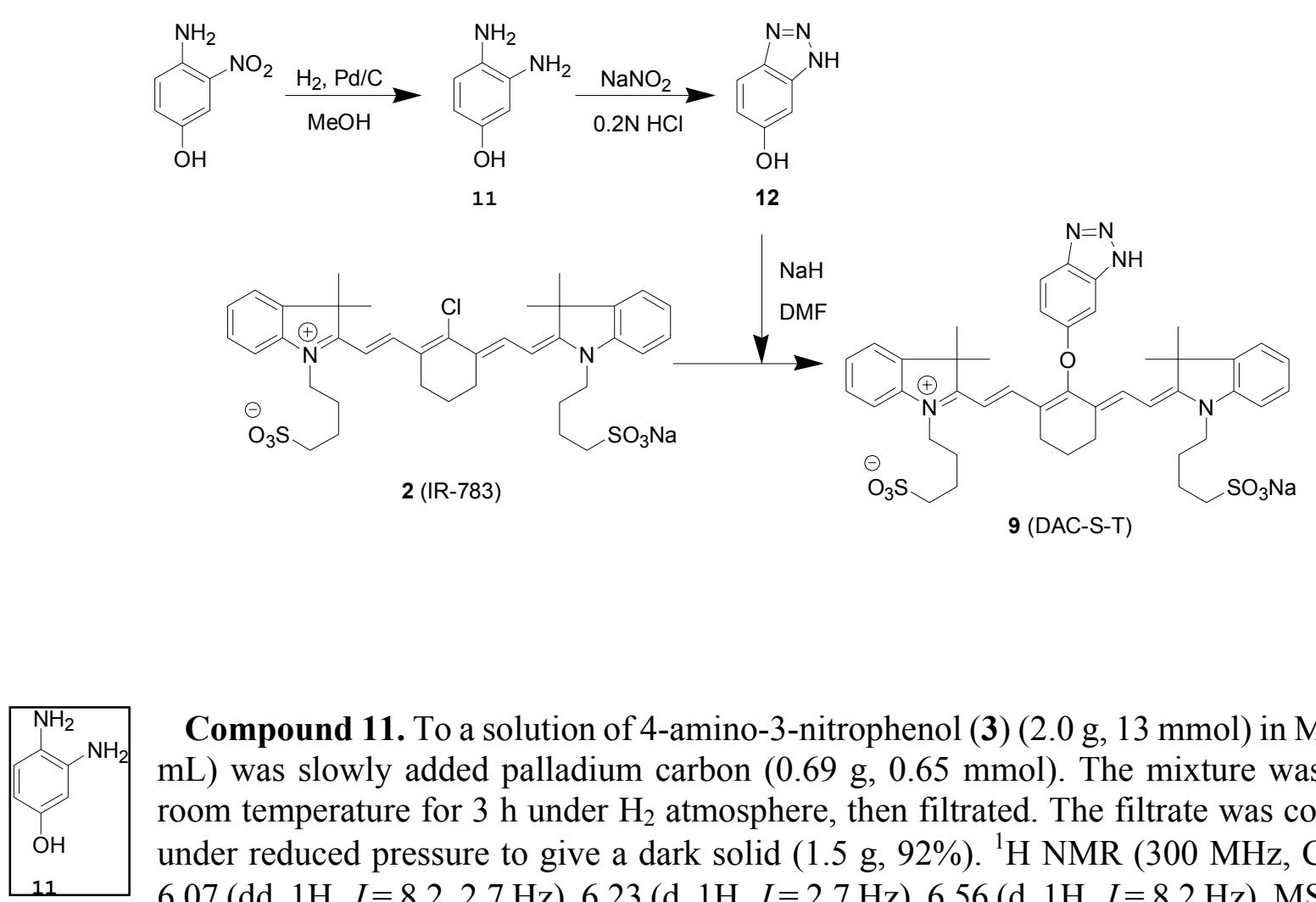

Compound 11. To a solution of 4-amino-3-nitrophenol (3) (2.0 g, $13 \mathrm{mmol})$ in $\mathrm{MeOH}$ (100 $\mathrm{mL})$ was slowly added palladium carbon $(0.69 \mathrm{~g}, 0.65 \mathrm{mmol})$. The mixture was stirred at room temperature for $3 \mathrm{~h}$ under $\mathrm{H}_{2}$ atmosphere, then filtrated. The filtrate was concentrated under reduced pressure to give a dark solid $(1.5 \mathrm{~g}, 92 \%) .{ }^{1} \mathrm{H}$ NMR $\left(300 \mathrm{MHz}, \mathrm{CD}_{3} \mathrm{OD}\right): \delta$ $6.07(\mathrm{dd}, 1 \mathrm{H}, J=8.2,2.7 \mathrm{~Hz}), 6.23(\mathrm{~d}, 1 \mathrm{H}, J=2.7 \mathrm{~Hz}), 6.56(\mathrm{~d}, 1 \mathrm{H}, J=8.2 \mathrm{~Hz}) . \mathrm{MS}\left(\mathrm{EI}^{+}\right): 124$ $\left(\mathrm{M}^{+}\right)$.

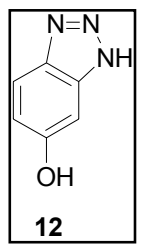

Compound 12. To a solution of $11(500 \mathrm{mg}, 4.0 \mathrm{mmol})$ in $0.2 \mathrm{~N} \mathrm{HCl}$ was slowly added $\mathrm{NaNO}_{2}(280 \mathrm{mg}, 4.0 \mathrm{mmol})$ at $0{ }^{\circ} \mathrm{C}$. The solution was stirred at room temperature for $1.5 \mathrm{~h}$, then neutralized with $2 \mathrm{~N} \mathrm{NaOH}$ and extracted with ethyl acetate. The organic extract was dried over $\mathrm{Na}_{2} \mathrm{SO}_{4}$, filtered and evaporated to give a brown solid $(440 \mathrm{mg}, 80 \%)$. ${ }^{1} \mathrm{H} \mathrm{NMR}$ $\left(300 \mathrm{MHz}, \mathrm{CD}_{3} \mathrm{OD}\right): \delta 6.88(\mathrm{~m}, 2 \mathrm{H}), 7.64(\mathrm{~d}, 1 \mathrm{H}, J=9.7 \mathrm{~Hz}) . \mathrm{MS}\left(\mathrm{EI}^{+}\right): 135\left(\mathrm{M}^{+}\right)$.

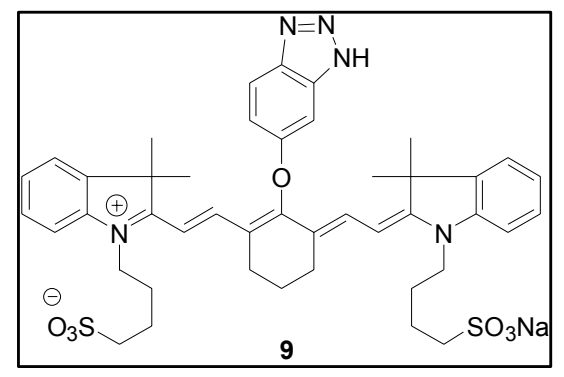

DAC-S-T (9). To a solution of 12 (34 $\mathrm{mg}, 0.25 \mathrm{mmol})$ in DMF (7 $\mathrm{mL})$ was added $\mathrm{NaH}(60 \%$ in mineral oil) $(10 \mathrm{mg}, 0.25 \mathrm{mmol})$. The mixture was stirred at room temperature for $10 \mathrm{~min}$ under an argon atmosphere, then a solution of IR-783 (2) (74 $\mathrm{mg}, 0.10 \mathrm{mmol})$ in anhydrous DMF $(2 \mathrm{~mL})$ was added via a syringe. The resulted reaction mixture was further stirred for $6 \mathrm{~h}$. Then, the solvent was removed under reduced pressure and the crude product was purified by silica gel chromatography with $10 \% \mathrm{H}_{2} \mathrm{O}$ in $\mathrm{CH}_{3} \mathrm{CN}$ to afford the crude product. Further purification was performed with semi-preparative HPLC to give a pure product as a green solid. (15 mg, 18\%). mp 234-236 ${ }^{\circ} \mathrm{C} .{ }^{1} \mathrm{H}$ NMR (300 MHz, DMSO- $\left.d_{6}\right): \delta 1.17(\mathrm{~s}, 12 \mathrm{H}), 1.70-1.97(\mathrm{~m}, 10 \mathrm{H})$, $2.48(\mathrm{~m}, 4 \mathrm{H}), 2.76(\mathrm{t}, 4 \mathrm{H}, J=6.0 \mathrm{~Hz}), 4.12(\mathrm{t}, 4 \mathrm{H}, J=6.6 \mathrm{~Hz}), 6.25(\mathrm{~d}, 2 \mathrm{H}, J=14.2 \mathrm{~Hz}), 7.13-7.48(\mathrm{~m}$, $10 \mathrm{H}), 7.81(\mathrm{~d}, 2 \mathrm{H}, J=14.2 \mathrm{~Hz}), 8.06(\mathrm{~d}, 1 \mathrm{H}, J=9.0 \mathrm{~Hz}) . \mathrm{MS}\left(\mathrm{FAB}^{+}\right): 826\left(\mathrm{M}-\mathrm{Na}^{+}+2 \mathrm{H}^{+}\right), 848\left(\mathrm{M}+\mathrm{H}^{+}\right)$, $870\left(\mathrm{M}+\mathrm{Na}^{+}\right)$. HRMS $\left(\mathrm{ESI}^{+}\right)$: calcd 870.2947, found 870.2952 $\left(\mathrm{M}+\mathrm{Na}^{+}\right)$. HPLC (eluent A/B = 50/50): $12.4 \mathrm{~min}$. 


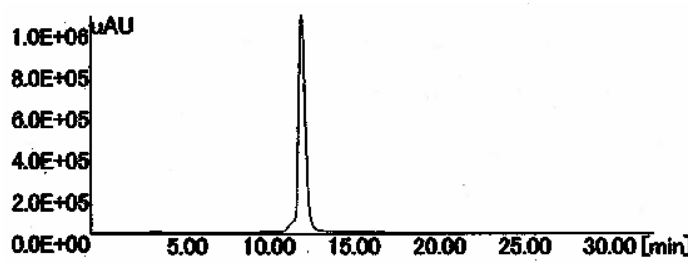

Table S1. Spectral Properties of DACs, DAC-Ts, and Indocyanine Green (ICG) ${ }^{\text {a }}$

\begin{tabular}{c|cccc}
\hline compound & $\begin{array}{c}\text { Absorbance max } \\
(\mathrm{nm})\end{array}$ & $\begin{array}{c}\text { Extinction } \\
\text { coefficient }\left(\times 10^{5}\right. \\
\left.\mathrm{M}^{-1} \mathrm{~cm}^{-1}\right)\end{array}$ & $\begin{array}{c}\text { Emission max } \\
(\mathrm{nm})\end{array}$ & $\begin{array}{c}\text { Relative quantum } \\
\text { yield }^{\mathrm{b}}\left(\times 10^{-2}\right)\end{array}$ \\
\hline DAC-P & 767 & 1.6 & 782 & 0.3 \\
DAC-P-T & 767 & 1.8 & 783 & 3.9 \\
DAC-S & 767 & 1.9 & 785 & 0.4 \\
DAC-S-T & 768 & 1.6 & 785 & 5.7 \\
ICG & 780 & 1.9 & 802 & 2.5 \\
\hline
\end{tabular}

${ }^{\mathrm{a}}$ All data were measured in $0.1 \mathrm{M}$ sodium phosphate buffer, $\mathrm{pH} 7.4(<0.6 \%$ DMSO as a cosolvent $)$.

${ }^{\mathrm{b}}$ Quantum yields of fluorescence were determined using that of ICG (0.13) in DMSO as a standard.

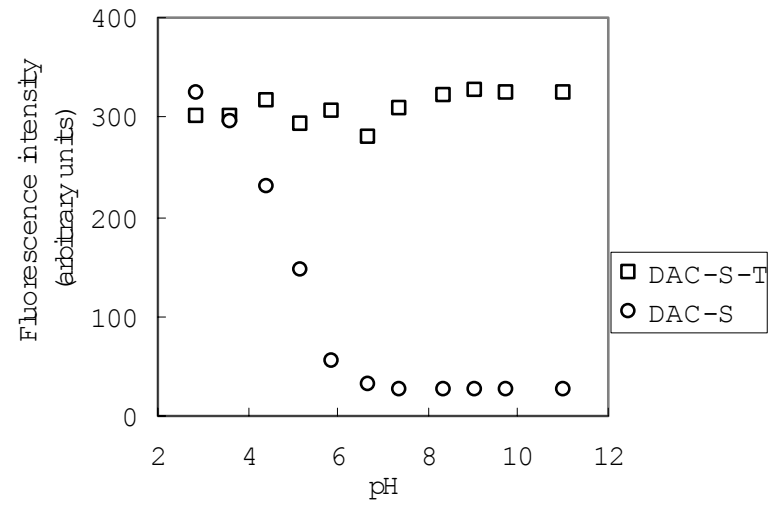

Figure S1. Effect of $\mathrm{pH}$ on the fluorescence intensity of DAC-S and DAC-S-T in $0.1 \mathrm{M}$ sodium phosphate buffer. The fluorescence intensities of DAC-S and DAC-S-T $(0.3 \mu \mathrm{M})$ were determined at 785 $\mathrm{nm}$ with excitation at $750 \mathrm{~nm}$. 


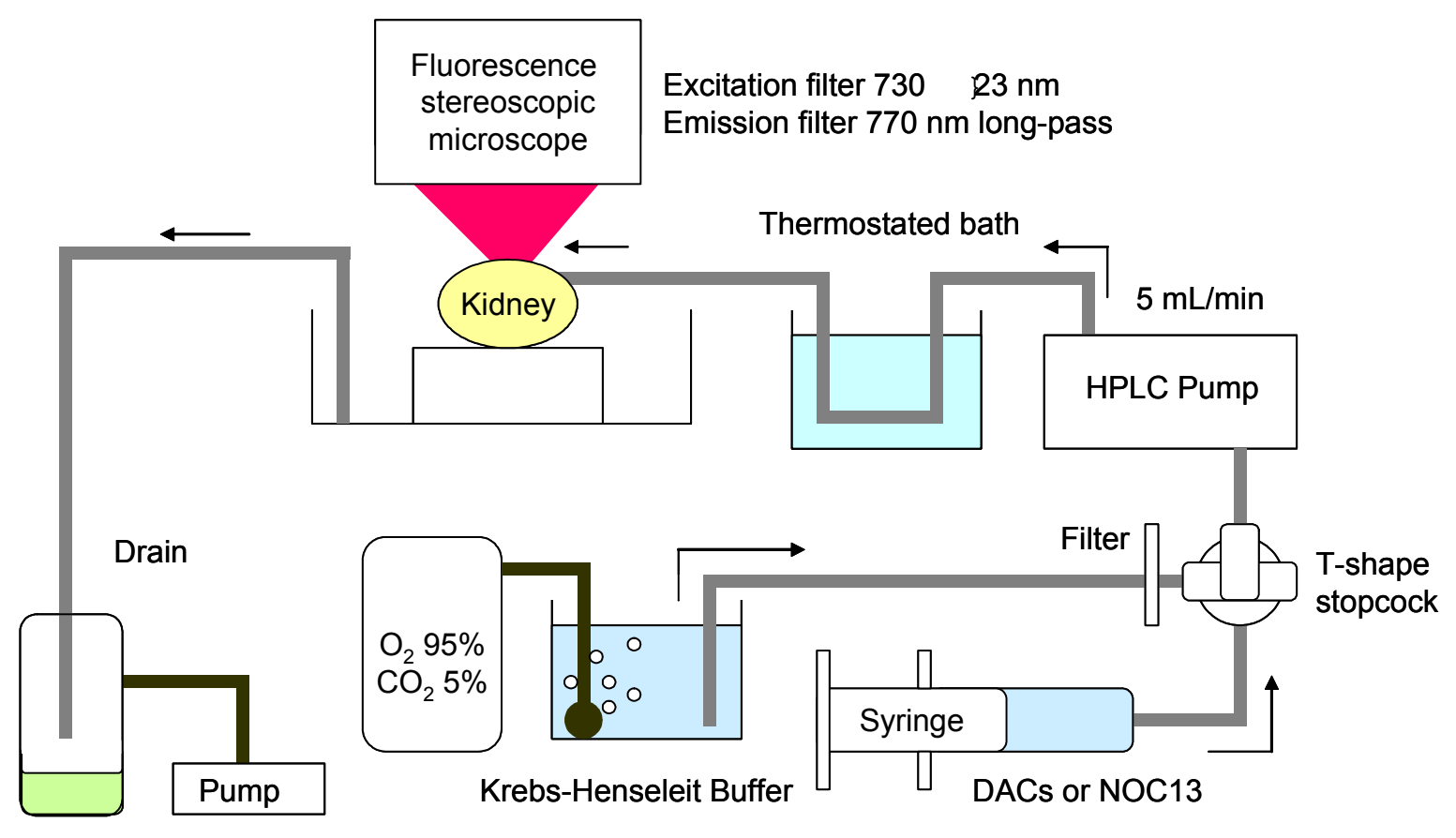

Figure S2. Diagram of the perfused rat kidney system

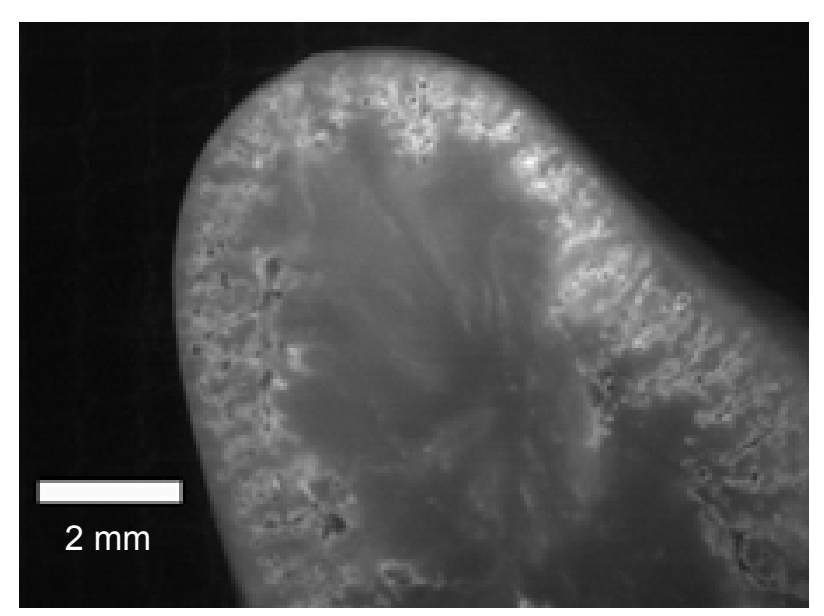

Figure S3. NIR fluorescence image of a renal section 